Original Article

\title{
EFFECT OF DIABETES MELLITUS ON RIFAMPICIN PEAK SERUM CONCENTRATION
}

\author{
SARANYA P.1, a, PARTHASARATHY V.*2, HARIPRASAD B. ${ }^{1}$, SHOBHA RANI H. ${ }^{3}$
}

1Sri Ramachandra University, No.1, Ramachandra Nagar Porur, Chennai, Tamil Nadu 600116, aSchool of Pharmaceutical Sciences, Vels University, Pallavaram, Chennai, ${ }^{2}$ Annamalai University, Annamalai Nagar, Chidambaram, Tamil Nadu 608002, ${ }^{3} \mathrm{Al}-\mathrm{Ameen}$ College of Pharmacy, Opp. Lalbagh Main Gate, Hosur Road, Bengaluru, Karnataka 560027

Email: vapartha@yahoo.com

Received: 03 Jun 2016 Revised and Accepted: 12 Aug 2016

ABSTRACT

Objective: To comparatively analyze the peak serum concentration ( $\mathrm{Cmax}$ ) of rifampicin and to determine the incidence of decreased Cmax between diabetic and non-diabetic adult pulmonary tuberculosis patients.

Methods: A cross-sectional observational study was carried out in the chest and tuberculosis (TB) department of a tertiary care hospital after the approval of the institutional ethics committee. Five millilitre (ml) of blood was withdrawn by venipuncture from each patient at a time point of $2 \mathrm{~h}$ post dose administration at steady state concentration $\left(\mathrm{C}_{\mathrm{ss}}\right)$. The separated serum was centrifuged at a rate of 3500 rotations per minute (rpm) for a period of fifteen minutes and the resultant serum was stored at-70 ${ }^{\circ} \mathrm{C}$ until analysis. Estimation of rifampicin concentration was carried out in Thermo TSQ Ultra (MS/MS) with Shimadzu 20 AD UFLC LC-MS.

Results: The mean (Standard Deviation (SD)) age of the study population was 46.8 (14.2) years. The mean serum $\mathrm{C}_{\text {max }}$ of rifampicin was significantly less in diabetic patients with pulmonary tuberculosis $(\mathrm{p}=0.0305)$. Statistically, a significant difference in the incidence of a decrease in $\mathrm{C}_{\text {max }}$ was found between diabetic and non-diabetic patients $(\mathrm{p}=0.0335)$. Diabetes mellitus was found to be the predominant factor that affects rifampicin $\mathrm{C}_{\text {max }}$.

Conclusion: In this study, an effect of diabetes mellitus (DM) on the peak serum concentration of rifampicin was observed. Patients with hyperglycemia levels had significantly reduced levels of rifampicin serum concentrations, thus showing an inversely proportional relationship between blood glucose and rifampicin serum levels.

Keywords: Rifampicin, $\mathrm{C}_{\max }$, Diabetes mellitus, Pulmonary tuberculosis, Diabetes and tuberculosis, DM and TB

(C) 2016 The Authors. Published by Innovare Academic Sciences Pvt Ltd. This is an open access article under the CC BY license (http://creativecommons. org/licenses/by/4. 0/) DOI: http://dx.doi.org/10.22159/ijpps.2016v8i10.13238

\section{INTRODUCTION}

The "captain of all these men of death", tuberculosis (TB) has been a menace to the humankind since the ancient time. TB-a serious and highly infectious disease caused by mycobacterium tuberculosis is the second leading cause for the high incidence of mortality rates worldwide. TB affects the lungs (Pulmonary tuberculosis-PTB) more commonly than the other parts including pleura, central nervous system, lymphatic system, bone and joints (extra-pulmonary tuberculosis). Hemoptysis, night sweats, loss of appetite and weight loss are the common symptoms of PTB $[1,2]$.

India is the country of highest $\mathrm{TB}$ and diabetes mellitus (DM) burden $[3,4]$. According to the world health organization (WHO) 2014 estimates, incidence of 2.5 million cases of active TB was reported to be in India out of 9.6 million cases incident globally. It also adds that "about $40 \%$ of the Indian population and one-third of the world's population are infected with the mycobacterium tuberculosis, where most of them have a latent infection rather than the active disease" $[5,6]$.

TB is referred as the top infectious killer disease worldwide [7]. In India, therapeutic success is achieved only in $88 \%$ of the treated TB patients, with $4 \%$ death, $2 \%$ treatment failure and $6 \%$ treatment default in patients on anti-tubercular therapy (ATT) [8]. TB is extremely associated with other comorbid conditions like diabetes mellitus, HIV/AIDs, multi-drug and extreme drug resistance [8]

The WHO recommends combination therapies with isoniazid rifampicin, ethambutol, pyrazinamide, streptomycin and other second line agents in intensive and continuous phases for the treatment of pulmonary tuberculosis [9]. Being highly bactericidal, rifampicin forms the nucleus of anti-tubercular therapy. However, the drug is associated with high inter-individual variability, and various clinical, environmental and genetic factors tend to alter the pharmacokinetic profile of rifampicin [10].
Diabetes mellitus has been reported to influence oral rifampicin pharmacokinetics by causing variability in absorption leading to decrease the systemic bioavailability of the drug [11]. Decreased systemic exposure to the drug leads to the emergence of multi-drug resistant bacilli and/or treatment failure [12]. Although several studies have been reported on the pharmacokinetics of rifampicin in tuberculosis patients, the impact of diabetes as a comorbid disease, on the peak serum rifampicin concentration is not addressed in India. Hence this study was carried out with an objective to determine the effect of diabetes mellitus on the peak serum concentration of rifampicin.

\section{MATERIALS AND METHODS}

\section{Method}

The study was carried out as a cross-sectional observational study in the chest and TB department of a tertiary care hospital. The study protocol was approved by the institutional ethics committee, Sri Ramachandra University (IEC/12/MAR/94/09). The study participants were recruited after obtaining written informed consent. All PTB patients between ages eighteen and sixty-five of either gender were included in the study. Pregnant females with tuberculosis, tuberculosis patients of both genders who were retropositive were excluded from the study.

\section{Serum collection and storage}

Five $\mathrm{ml}$ of blood was withdrawn by venipuncture from each patient at a time point of $2 \mathrm{~h}$ post dose administration at steady state $\left(\mathrm{C}_{\mathrm{ss}}\right)$. The blood samples were collected in serum separator vacutainers containing clot activator and allowed to stand for $30 \mathrm{~min}$. The vacutainers were shifted to a laboratory for centrifugation after packing with ice bags containing silica gel. The separated serum was centrifuged at a rate of 3500 rotations per minute (rpm) for a period of fifteen minutes, and the resultant serum was stored at- $70^{\circ} \mathrm{C}$ until analysis. 


\section{Estimation of rifampicin concentration (Srivastava et al., 2012) [13]}

The frozen samples were thawed at room temperature, and an aliquot of $200 \mu \mathrm{l}$ sample was transferred to pre-labeled Ria vials. 50 $\mu \mathrm{l}$ of internal standard (Roxithromycin $1.000 \mu \mathrm{g} / \mathrm{ml}$ ) was added and vortexed well-using cyclomixer. $0.400 \mathrm{ml}$ of $100 \%$ acetonitrile was added, and the capped vials were re-vortexed in vibramax at 2000 rpm for $10 \mathrm{~min}$ followed by centrifugation at $4500 \mathrm{rpm}$ for $10 \mathrm{~min}$ at $4{ }^{\circ} \mathrm{C}$. $0.300 \mathrm{ml}$ of supernatant was transferred into pre-labeled injector vials and loaded into LC-MS autosampler. Estimation of rifampicin concentration was carried out in Thermo TSQ Ultra (MS/MS) With Shimadzu 20 AD UFLC LC-MS. ZORBAX Eclipse Plus C18 column of dimensions $4.6 \mathrm{mmx}, 150 \mathrm{~mm}, 5 \mu \mathrm{m}$ and acetonitrile $10 \mathrm{mmol}$, ammonium acetate $(80: 20 \% \mathrm{v} / \mathrm{v})$ were used as stationary and mobile phases respectively at a flow rate of $1 \mathrm{ml} /$ minute.

\section{Statistical analysis}

All statistical analyses were performed using SPSS 17.0 and Graph pad prism 7.0. Pearson's correlation was used to determine the linear dependency of Cmax on individual covariates. Chi-square analysis was used to determine the effect of dichotomous categorical variables. Unpaired student t-test was used to compare two groups. A p-value less than 0.05 were considered statistically significant throughout the study $(95 \% \mathrm{CI})$.

\section{RESULTS}

Forty-five patients who fulfilled the inclusion criterion were recruited in the study. Twenty-five patients were non-diabetic whereas 20 patients were diabetic. The mean (SD) age of the study population was $46.8(14.2)$ years. The study participants were constituted of $68.88 \%$ male and $31.12 \%$ female (fig. 1). The distribution of study population based on their age group is depicted in table 1.

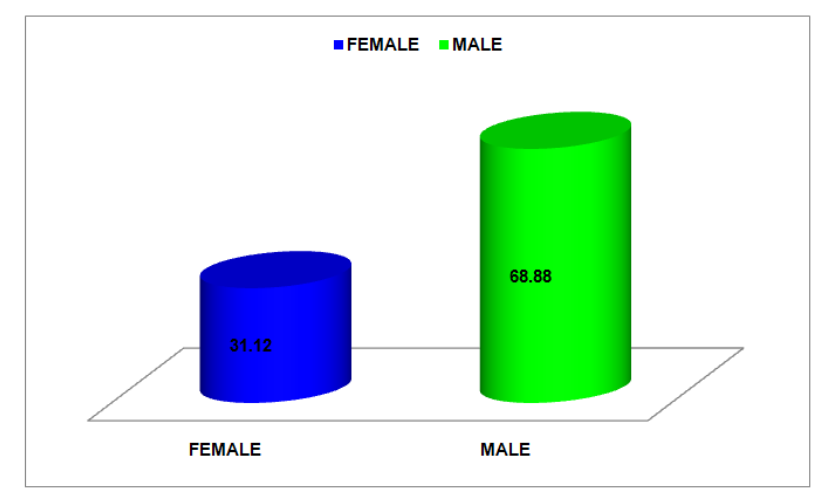

Fig. 1: Gender wise distribution

Table 1: Age-wise distribution

\begin{tabular}{lll}
\hline Age & No. of patients $\mathbf{N}=\mathbf{4 5}$ & Percentage $\mathbf{( \% )}$ \\
\hline $21-30$ & 16 & 35.5 \\
$31-40$ & 11 & 26.5 \\
$41-50$ & 7 & 13.5 \\
$51-60$ & 9 & 20 \\
$>61$ & 2 & 4.5 \\
\hline
\end{tabular}

The mean (SD) age of males was comparatively higher than that of females with values of 48.7 (10.6) and 43.1 (11.2) respectively $(\mathrm{P}=0.532)$. The mean age of non-diabetic TB patients was comparatively higher than that of diabetic patients with values of 46.6 (8.4) and 42.4 (9.2) respectively.

The body weight of about $86.6 \%$ of the patients was less than $50 \mathrm{~kg}$ and $13.4 \%$ of patients was greater than $50 \mathrm{~kg}$. No statistically significant difference in body weight was found between diabetic and non-diabetic patients $(\mathrm{P}=0.5440)$. About $51.12 \%$ of the patients were smokers and $48.88 \%$ were non-smokers. The most commonly prescribed concomitant medications were ranitidine and aluminum hydroxide (36\%). The other co-medications prescribed were insulin $(34 \%)$, paracetamol $(18 \%)$, theophylline $(4 \%)$ and supplements $(8 \%)$. The distribution of study participants based on the RNTCP (revised national tuberculosis control program) treatment (CAT I and CAT II) they received is shown in fig. 2.

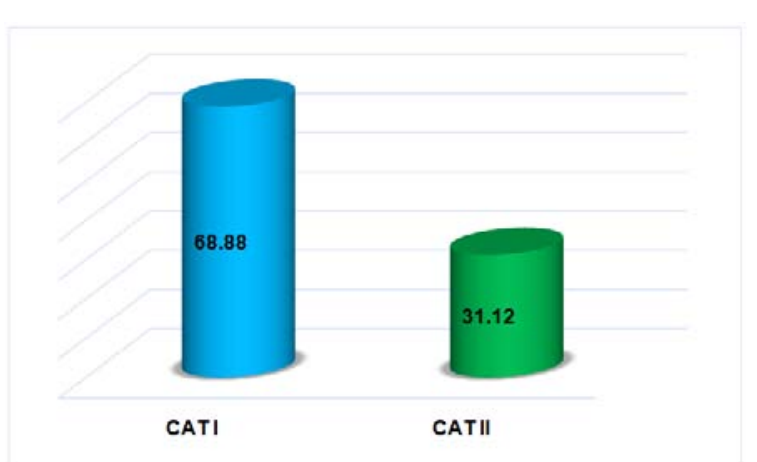

Fig. 2: Distribution based on CAT regimen

Around $55.55 \%$ were found to be alcoholic and $44.45 \%$ were nonalcoholic. Nearly $68.8 \%$ patients had a prior history of TB whereas $31.2 \%$ patients had no history of TB. Twenty-five (58\%) patients of the study population were non-diabetic, and 20 (42\%) were diabetic. Almost $83 \%$ of the diabetic patients were on a combination of insulin and metformin. Very few patients received the other oral hypoglycemic medications.

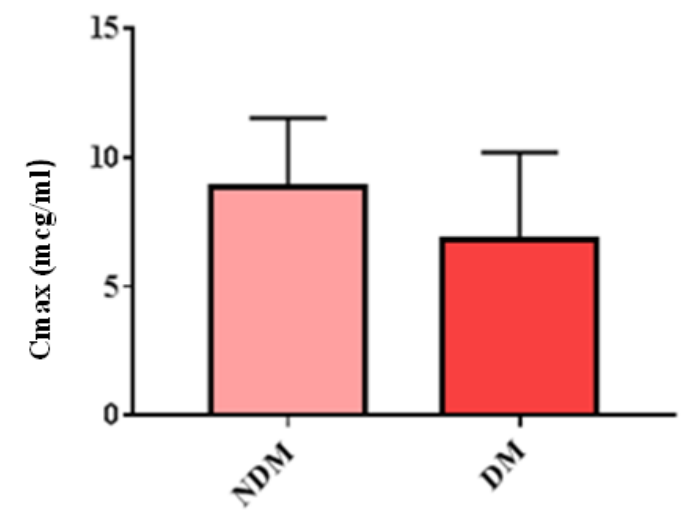

Fig. 3: Comparison of peak serum rifampicin concentration in diabetic (DM) and nondiabetic patients (NDM) with pulmonary tuberculosis $(\mathrm{P}=\mathbf{0 . 0 3 0 5})$

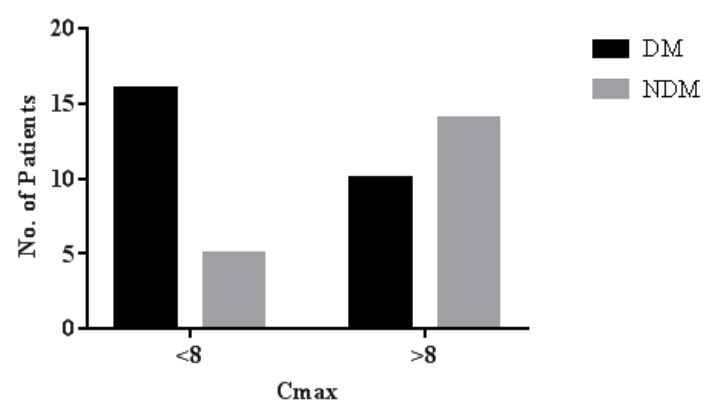

Fig. 4: Incidence of sub-therapeutic peak serum rifampicin concentration between diabetic and non-diabetic patients with pulmonary tuberculosis $(\mathrm{P}=\mathbf{0 . 3 3 5})$ 
The mean serum $\mathrm{C}_{\max }$ of rifampicin was significantly less in diabetic patients with pulmonary tuberculosis $(\mathrm{P}=0.0305)$ as shown in fig. 3 . Statistically, a significant difference in the incidence of a decrease in $\mathrm{C}_{\max }$ was found between diabetic and non-diabetic patients $(\mathrm{P}=0.0335)$ as depicted by fig. 4 .

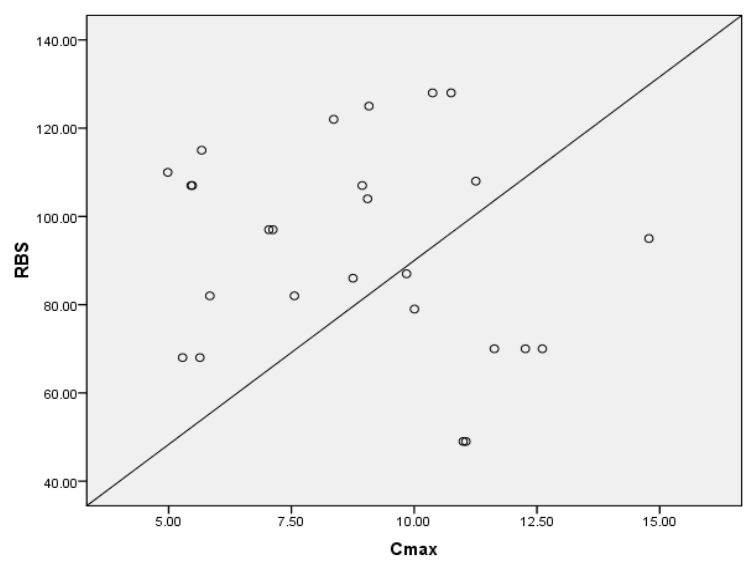

Fig. 5: Correlation of random blood sugar (RBS) and peak rifampicin serum concentration in pulmonary tuberculosis patients without diabetes

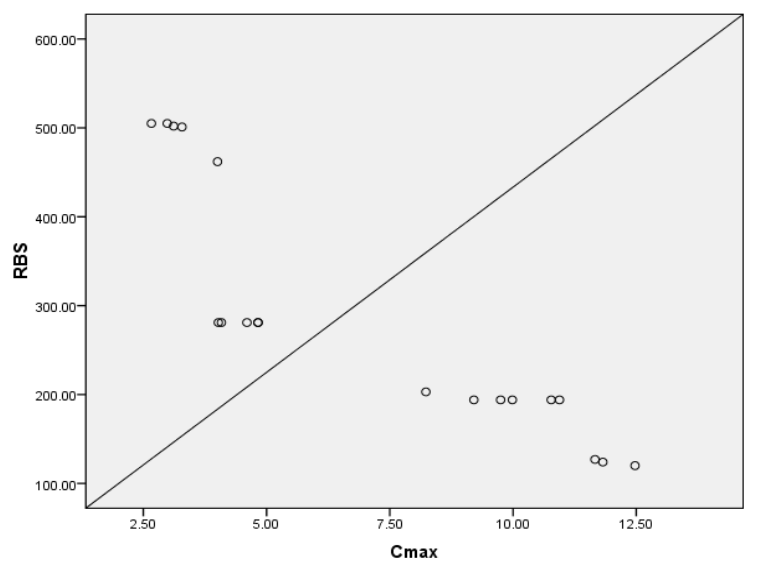

Fig. 6: Correlation of random blood sugar and peak rifampicin serum concentration in pulmonary tuberculosis patients with diabetes

The values of RBS and observed $\mathrm{C}_{\max }$ of diabetic and non-diabetic patients with pulmonary tuberculosis were correlated using Pearson's correlation. The correlation between RBS and rifampicin Cmax in pulmonary tuberculosis patients without diabetes is shown in fig. 5. The fig. 6 depicts the correlation between RBS and rifampicin Cmax in pulmonary tuberculosis patients with diabetes.

\section{DISCUSSION}

This study has examined the effect of diabetes on the peak serum rifampicin concentration in patients with pulmonary tuberculosis, by comparing the expected rifampicin Cmax of patients with PTB only. Peak serum rifampicin concentrations tend to be decreased in pulmonary tuberculosis patients with comorbidity diabetes when compared to PTB patients without diabetes. This is in line with the study carried out by Nijland et al. (2006) [12]. The mean age (48.7 y) of the male patients was greater when compared to a similar study conducted by Mendez (2012) [14]. The mean age of PTB patients with diabetes and without diabetes in the present study was in contrast to that conducted by Ruslami et al. (2010) [15]. However, our results were similar to Ruslami et al. when the statistical significance in body weight between these groups was analyzed [15]. Exposure to rifampicin has been reported to be two-fold lower in diabetic than in non-diabetic patients with tuberculosis during the continuation phase of treatment [14]. Diabetes mellitus was found to be the predominant factor that affects rifampicin $\mathrm{C}_{\max }$. Clinical studies suggest that hyperglycemic state decreases the release of gastric acid from parietal cells and thereby increases gastric $\mathrm{pH}$. Being highly $\mathrm{pH}$ dependent for solubility and well absorbed from an acidic $\mathrm{pH}$, a shift in the gastric $\mathrm{pH}$ due to hyperglycemia delays rifampicin absorption and thereby tend to prolong the $t_{\max }$ and decrease the $C_{\max }$ [15]. In addition, gastrointestinal ailments such as gastroparesis which are common in chronic diabetics may either delay or impair absorption of rifampicin leading to decreased systemic availability [17]. Univariate analyses were performed to determine the relationship between random blood glucose and rifampicin $\mathrm{C}_{\max }$. A near inverse correlation was obtained in diabetic patients (Pearson's correlation $=-0.402, \mathrm{P}$ value $=0.001$ ) ; however such a correlation was not observed in non-diabetic pulmonary tuberculosis patients suggesting that the drug absorption is disfavoured only under hyperglycemic conditions and linear relationship does not exist between blood glucose and rifampicin $\mathrm{C}_{\max }$ (Pearson's correlation $=0.006$, $\mathrm{P}$ Value $=0.962$ ). This is similar to the study reported by Nijland et al. (2006), Heysell et al. (2010) and Heysell et al. (2013) [12,16-17]. The RBS vs $\mathrm{C}_{\max }$ correlation plot of non-diabetic and diabetic PTB patients is shown in fig. 3 and 4 respectively. In addition, the incidence of decreased $\mathrm{C}_{\max }$ was found to be high in PTB patients with diabetes than patients without diabetes $(\mathrm{P}<0.0001)$. These findings suggest that exposure to rifampicin is decreased in PTB patients with comorbid diabetes mellitus, which is in line with that reported by Nijland et al. (2006) [12]. Further, linear relationship observed between RBS and $\mathrm{C}_{\max }$ in diabetic patients was not observed in non-diabetic patients suggesting that decreased in exposure is a direct result of hyperglycemic state and not due to diabetes mellitus as a disease. Further prospective studies to determine the pharmacokinetic profile of rifampicin in PTB patients with diabetic control are required to ascertain whether serum concentrations of rifampicin remain unaltered.

These findings suggest that absorption of rifampicin is decreased due to increase in gastric $\mathrm{pH}$ caused by elevated blood glucose level. Therefore, rifampicin administered orally 1-2 $\mathrm{h}$ after a carbohydrate-rich meal may not be effectively absorbed. Thus, rifampicin has to be taken in an empty stomach for effective absorption both in diabetic and non-diabetic patients. These findings have to be confirmed in studies involving larger populations.

A sample size of the study population was smaller, the effect of other factors including unexplained variability in peak serum rifampicin concentrations, the effect of co-administered drugs, the role of genetic polymorphism in low rifampicin concentration was not studied and hence were the limitations of the study.

\section{CONCLUSION}

In this study, an effect of DM on the peak serum concentration of rifampicin was observed. Patients with hyperglycaemic levels had significantly reduced levels of rifampicin serum concentrations, thus showing an inversely proportional relationship between blood glucose and serum rifampicin levels. However, only the blood glucose levels and body weight were correlated with the concentration of rifampicin, further studies need to be carried out in a larger population in order to rule out the various factors which affect the drug absorption of rifampicin in pulmonary tuberculosis patients with comorbid diabetes.

\section{CONFLICT OF INTERESTS}

\section{Declared none}

\section{REFERENCES}

1. Fogel N. Tuberculosis: a disease without boundaries. Tuberculosis 2015;95:527-31.

2. Niazi AK, Kalra S. Diabetes and tuberculosis: a review of the role of optimal glycemic control. J Diabetes Metab Disord 2012;11:1-4.

3. TB Facts. TB, tests, drugs, statistics [Internet]. TB Facts. Org; 2016. Available from: http://www.tbfacts.org/. [Last accessed on 18 Apr 2016]. 
4. Preethi S, Pradeep K, Rachna S, Vijaykumar A. Futuristic scope of biomarkers in tuberculosis. Asian J Pharm Clin Res 2015;8:34-6.

5. Sutrisna EM. Autoinduction properties of rifampicin on Javanese tuberculosis with variant type CYP3A4*1G. Asian J Pharm Clin Res 2015;8:21-3.

6. Tuberculosis [Internet]. World Health Organization; 2016. Available from: http://www.who.int/mediacentre/ factsheets/ fs104/en/. [Last accessed on 18 Apr 2016].

7. TB India 2015 Revised National TB Control Programme Annual Status Report, New Delhi; 2015. Available from: www.tbcindia.nic.in. [Last accessed on 18 Apr 2016].

8. Marais BJ, Lönnroth K, Lawn SD, Migliori GB, Mwaba P, Glaziou $\mathrm{P}$, et al. Tuberculosis comorbidity with communicable and noncommunicable diseases: integrating health services and control efforts. Lancet Infect Dis 2013;13:436-48.

9. Morton R. Phthisiolgia: or a treatise of consumptions. London: Smith and Walford; 1694. p. 28

10. WHO Annual Report. Tuberculosis control in South-East-Asia Region ISBN 978-92-9022-476-1 (NLM classification: WU 30); 2010.

11. PV Rao. Persons with type 2 diabetes and comorbid active tuberculosis should be treated with insulin. Int J Diabetes 1999;19:1-4.

12. Nijiland H, Ruslami R, Stalenhoef JE, Nelwan EJ, Alisjahbana B, Nelwan $\mathrm{RHH}$, et al. Exposure to rifampicin is strongly reduced in patients with tuberculosis and type 2 diabetes. CID 2006;43:848-54.
13. Srivastava A, Waterhouse D, Ardrey A, Ward SA. Quantification of rifampicin in human plasma and cerebrospinal fluid by a highly sensitive and rapid liquid chromatographic-tandem mass spectrometric method. J Pharm Biomed Anal 2012;70:523-8.

14. Mendez AR, Davies G, Ardrey A, Jave O, Romero SL, Ward SA, et al. Pharmacokinetics of rifampicin in Peruvian tuberculosis patients with and without comorbid diabetes or HIV. Antimicrob Agents Chemother 2012;56:2357-63.

15. Ruslami R, Nijiland HMJ, Adhiarta GN, Kariadi HKS, Alisjahbana B. Pharmacokinetics of anti-tuberculosis drugs in pulmonary tuberculosis patients with type 2 diabetes. Antimicrob Agents Chemother 2010;5:1068-74.

16. Heysell SK, Moore JL, Keller SJ, Houpt ER. Therapeutic drug monitoring for slow response to tuberculosis treatment in a state control program, Virginia, USA. Emerging Infectious Diseases 2010;16:1546-53.

17. Heysell SK, Moore JL, Staley D, Dodge D, Houpt ER. Early therapeutic drug monitoring for isoniazid and rifampin among diabetics with newly diagnosed tuberculosis in Virginia, USA. Tuberculosis Res Treatment 2013;23:1-6.

\section{How to cite this article}

- Saranya P, Parthasarathy V, Hariprasad B, Shobha Rani H. Effect of diabetes mellitus on rifampicin peak serum concentration. Int J Pharm Pharm Sci 2016;8(10):149-152. 